\title{
Multiplication of Matrices
}

A method for finding rapidly the product of two matrices has been developed at the Ballistic Research Laboratory, Aberdeen Proving Ground. The method uses punched cards and a new machine built by the IBM Corporation for the Laboratory.

Each element of each matrix is punched on a separate card together with its row and column number and other identifying material. The cards representing matrix $A$ are sorted by rows and placed in one of the two feed hoppers of the machine, the cards of matrix $B$ are sorted by columns and placed in the other feed hopper. The machine, if properly wired, will feed cards from both hoppers, take the product of the two numbers which it reads out of the two cards (at the same time comparing the column index of the $A$ card with the row index of the $B$ card, and stopping if by some mistake these two indices are not equal), and accumulate the products as long as these indices increase. After the first $n$ cards (i.e. when the compared indices drop from $n$ to 1 ) it will store the number accumulated up to this time (which is $c_{11}$, the first element of the product matrix $C=A B$ ), start accumulating anew and punch $c_{11}$ on the next card of the $B$ stack into an unused part of the card. In the same way the machine computes and punches $c_{28}, \cdots, c_{n n}$.

Before beginning the operation, the last $n$ cards (representing the $n$th column) of $B$ are duplicated and placed at the end of the stack. After $n^{2}$ cards have passed through the machine, or at any earlier time, the cards which have passed through are taken out of their stackers and put back into the feed hoppers. Because of the extra $n$ cards in $B$, the two stacks are now out of phase by $n$, and the machine, without any change in wiring or without even stopping, computes $c_{1, n}, c_{21}, c_{23}, \cdots, c_{m n-1}$. It punches each of these numbers "at the first opportunity," i.e. into the first card of $B$ which does not yet have a $c_{i j}$ punched into it. The process is repeated until all elements of $C$ are computed and punched. This is done when the $n^{2}+n$ cards of $B$ have passed through the machine $n$ times. (If the machine is left running at that time, it will continue to compute but will not punch any more results.) The $n^{2}+n$ cards of $B$ now contain all elements of $C$, the elements of the $n$th column appearing twice. Thus the cards can be used for another multiplication in which $C$ is the right-hand factor. The wiring is such that whenever the machine punches a number $c_{i j}$, it also punches the indices $i$ and $j$.

The time required for the operation varies approximately as the cube of $n$. For multiplying up to six significant figures the machine feeds 6,000 cards per hour; for more than six significant figures but not exceeding twelve, the rate is 3,000 per hour. Thus two 9-row matrices with elements of not more than six significant figures can be multiplied in about nine minutes (the significant figures of all elements should be in the same six decimal positions). Because of the necessity of sorting the cards, of checking and correcting errors, etc., the actual time required for the whole operation may well be several times as long as the calculated running time of the machine. This is partly due to the fact that the machine is still in its experimental stage. Even so, this method is believed to be considerably faster than any other method in use at this time. 
It is possible to set the machine in such a way that it will produce $k I-C$ instead of $C$. This is useful in finding the inverse $X$ of a matrix $A$ by a process of successive approximations such as $X_{n+1}=X_{n}\left(2 I-A X_{n}\right)$. (See H. Hotelling, "Some new methods in matrix calculation," Annals Math. Statistics, v. 14, 1943, p. 1f.)

The machine is not specifically designed for the purpose of multiplying matrices. It is a general purpose computer, capable of handling such problems as projective transformations, third order interpolations and others.

Ballistic Research Laboratory

Franz L. Alt Aberdeen Proving Ground, Md.

\section{RECENT MATHEMATICAI TABLES}

\section{3[A-E, G-I, K-N, U].-A. Fletcher, J. C. P. Miller, \& L. Rosenhead, An Index of Mathematical Tables, London, Scientific Computing Service, 23 Bedford Square, W.C. 1, 1946. viii, 450 p. $15.6 \times 25.3 \mathrm{~cm} .75$ shillings.}

Five years ago there were less than ten experts deeply versed in the international field of mathematical tables and their reliability. Of these experts, of ten responding to requests for information, one was L. J. Comrie, the publisher of the work under review. With war's advent research workers on its problems were on every hand clamoring for enlightenment as to the best tables, if any, of this or that function, of such and such a range of argument. At least four countries soon felt that something ought to be done to assemble and to disseminate tabular information.

The National Research Council of the United States was the first to effect some relief in this regard, by the foundation three years ago of the quarterly publication, in which this review appears, and which has recently completed its first volume of nearly 500 pages. England came next, in 1943, with first printers' proofs of the Index now before us, a work by three well-known members of the department of Applied Mathematics at the University of Liverpool. Assembly of material which later developed into the Index was begun some years before, and the publisher expected that the volume would be ready for distribution early in 1944, but insurmountable difficulties besetting English printers delayed publication for two years. On account of this delay, proofs were revised so as to take account of new material to the end of 1944. Thus we now have the first work of its kind. During the past year and a half the reviewer has become thoroughly familiar with this publication through checking of early proof and, practically the final proof, which is now before him. In our limited space it will be quite impossible adequately to suggest the extraordinary richness of this epochmaking work, filled with so many excellencies for guiding to his goal the searcher for existing printed or ms. tabular aid.

The work contains the following two main divisions: Part I, p. 18-372, Index according to Functions; and Part II, p. 373-444, Bibliography. We shall first consider the latter. Here is a complete list of the published material referred to in Part I, arranged alphabetically according to authors. In the case of books or pamphlets brief titles are given, but in the case of tables, or other articles, in serials, the titles of the articles are rarely given, but only other bibliographical details. Under each name the entries are arranged chronologically, so that the first entry is always the year of publication (a few exceptions which we shall not explain), then the name of the serial, the series, the volume number, and inclusive pages of the article containing the table or other material. When several entries were published in the same year, they are differentiated by the different entries; for example, under Airey we have five entries 1911a, 1911b, 1911c, 1911d, 1911e; and also 1918, 1918 a showing that with more than one entry for a given year the first entry is not necessarily associated with some letter. 